\title{
Digitale Lehre und Prüfung im Grundlagenfach Maschinenelemente
}

\author{
T. Rosenlöcher ${ }^{*}$, B. Schlecht \\ Lehrstuhl Maschinenelemente, Institut für Maschinenelemente und Maschinenkonstruktion, Fakultät Maschinenwesen, \\ Technische Universität Dresden
}

\begin{abstract}
In Vorlesungen, Übungen und auch Praktika steht neben der Vermittlung von Wissen mit Hilfe von Tafelbildern, Folien und Präsentationen das kontinuierliche Einholen einer Rückmeldung von den Studierenden im Mittelpunkt, über die man als Lehrender bewerten kann, inwieweit die Ausführungen durch die Zuhörer auch verstanden wurden. Im Seminarraum ist dies ohne Einschränkungen möglich. Bei großen Vorlesungen können in den meisten Fällen nur die ersten Reihen für ein solches Feedback einbezogen werden. In drei Semestern der Lehre über verschiedene digitale Wege musste schlagartig akzeptiert werden, dass eine Rückmeldung zu den Lehrveranstaltungen nicht direkt und zumeist erst mit Evaluationen am Ende des Semesters durch die Studierenden gegeben werden. Die Überführung bewährter Lernkonzepte in einen Lehrbetrieb ohne Präsenzunterricht unter den ungünstigen zeitlichen wie auch technischen Randbedingungen erforderte enorme Anstrengungen, erwies sich jedoch auch als eine wirksame Antriebskraft, die Art und Weise zu Lehren und die Bedürfnisse der Studierenden aus einem anderen Blickwinkel zu sehen.
\end{abstract}

In lectures, exercises and practical courses, the focus is not only on imparting knowledge with the help of blackboards, slides and presentations, but also on continuously obtaining feedback from the students, which the teacher can use to assess the extent to which the audience has understood the explanations. This is possible without restrictions in the seminar room. In large lectures, in most cases only the first rows can be included for such feedback. In three semesters of teaching via various digital channels, it had to be abruptly accepted that feedback by the students on courses is not given directly and mostly only with evaluations at the end of the semester. The transfer of proven learning concepts into a teaching mode without face-to-face teaching under the unfavourable time as well as technical boundary conditions required enormous efforts, but also proved to be an effective driving force to see the way of teaching and the needs of the students from a different perspective. 


\section{Einleitung}

Lehren ohne Hörsaal und Seminarraum über die Dauer mehrerer Semester! Diese Herausforderung stand vor dem Frühjahr des Jahres 2020 nie zur Diskussion. Wie auch in vielen anderen Kursen, bedarf die Vermittlung des Lehrinhaltes im Präsenzstudium eines abgestimmten Konzeptes zwischen Vorlesungen, Übungen und gegebenenfalls Praktika, was aufgrund der begrenzten zeitlichen Kapazitäten auch nicht immer gelingt. Die plötzliche Notwendigkeit, bewährte Unterrichtsformen in kürzester Zeit in einem vollkommen neuen Format bereitzustellen und dabei nur die vorhandenen und nicht dafür vorgesehenen technischen Möglichkeiten zu nutzen, erforderte im Frühjahr 2020 eine vollständige Umstellung des ansonsten gut eingespielten Semesterablaufes.

\section{Das Studienjahr in Präsenz}

Im zweiten Studienjahr werden den fast 500 eingeschriebenen Studentinnen und Studenten des Studienganges Maschinenbau im Rahmen der Vorlesungen (3 SWS) und Übungen (2 SWS) die Grundlagen im Fach Maschinenelemente vermittelt. Aufbauend auf der Festigkeitslehre lernen die Studierenden das Vorgehen zur Gestaltung und Dimensionierung von Wellen, Welle-Nabe-Verbindungen, Schrauben, Federn, Kupplungen, Wälzlagern, Gleitlagern und Verzahnungen kennen (Abb. 1).
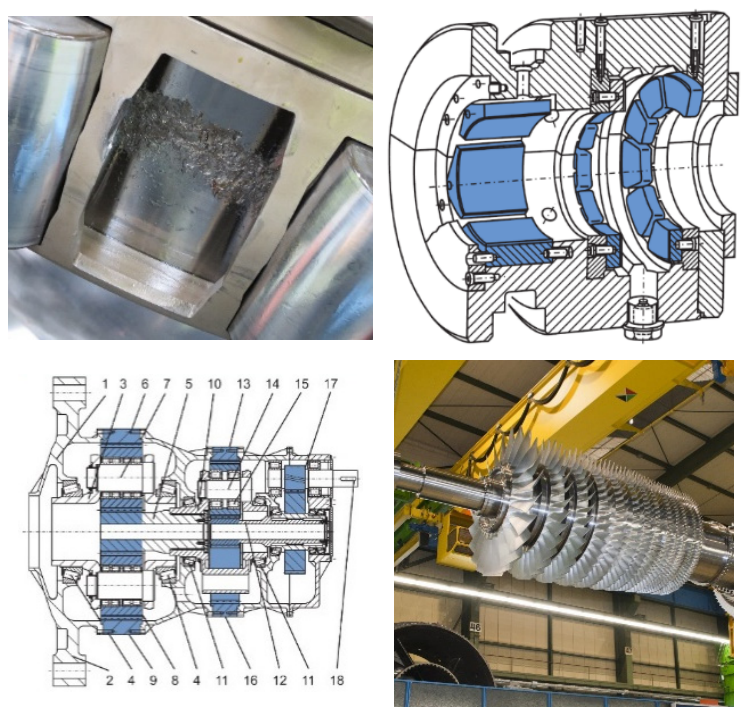

Abb. 1: Maschinenelemente - Einsatzbeispiele
Mit Hilfe von Präsentationen wird der Lehrinhalt den Studierenden erläutert und die Folien werden bei Bedarf durch Anmerkungen und Skizzen ergänzt. Die Unterlagen zur Vorlesung stehen in dem Lernmanagementsystem OPAL zum Herunterladen bereit. In den einzelnen Themengebieten wird anhand von verschiedenen Anwendungsbeispielen fortwährend der Bezug zur praktischen Anwendung des Erlernten hergestellt.

In den wöchentlichen Übungen werden die Auslegung und die Nachrechnung von Maschinenelementen anhand von verschiedenen Aufgaben und Anschauungsobjekten zu Beginn jeder Stunde ausführlich durch Mitarbeiter des Lehrstuhls erklärt und durch die Studierenden selbständig mit Hilfe der Übungsleiter und Tutoren geübt (Abb. 2).

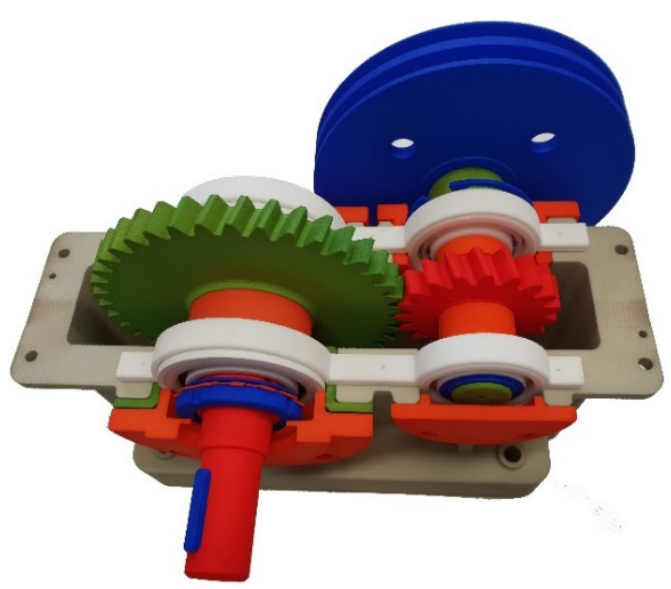

Abb. 2: Modell eines einstufigen Getriebes

Dabei dienen die Mitschriften der Studierenden zur Dokumentation des Lösungsweges, zur Prüfungsvorbereitung und zur Bearbeitung der Prüfungsaufgaben, da die Unterlagen während des Rechenteils der Prüfung genutzt werden können. Bei der Erläuterung der Übungsaufgaben werden die einzelnen Arbeitsschritte daher sukzessiv erklärt und aufgeschrieben, so dass beim eigenständigen Berechnen der Lösungen die Handhabung der Normen und Richtlinien geübt und die Ergebnisse dokumentiert werden können. Für die Übungen werden die 14 Seminargruppen in 12 Übungsgruppen eingeteilt, so dass Tutoren und Übungsleiter auf individuelle Fragestellungen der Studierenden eingehen können. In den Übungen erfolgt auch die Betreuung des Semesterbeleges, in dem die Studierenden 
selbständig auf Grundlage der zu berechnenden Lasten verschiedene Maschinenelemente auslegen, erforderliche Normteile auswählen und in einer Baugruppe zusammenführen müssen. Am Ende des Studienjahres sind dann technische Freihandzeichnungen und CADZeichnungen der Baugruppe und der Einzelteile sowie die Berechnungsunterlagen einzureichen.

Ergänzend zu den Vorlesungen und Übungen, wird in jedem Sommersemester das Vorrechnen angeboten. In neun Veranstaltungen werden weitere Übungsaufgaben für alle Studierenden im Hörsaal Schritt für Schritt gelöst und der Rechenweg sowie die Handhabung von Formeln, Tabellen und Schaubildern ausführlich erläutert (Abb. 3). Auch die während der Lehrveranstaltung selbständig erstellten Mitschriften sollen als Grundlage und zur Vorbereitung der Prüfung dienen.

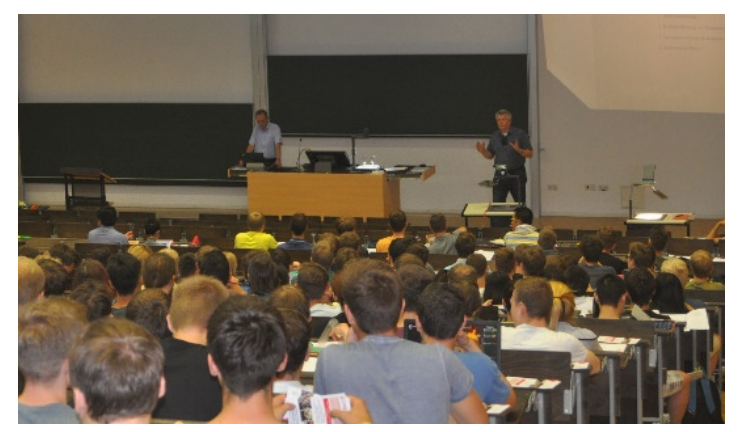

Abb. 3: Vorrechnen im Fach Maschinenelemente

Für die Studiengänge Mechatronik, Regenerative Energiesysteme, Wirtschaftswissenschaft und Textil- und Konfektionstechnik wird der Lehrinhalt des Faches Maschinenelemente in komprimierter Form vermittelt. Dazu werden wöchentliche Vorlesungen und Übungen angeboten. Die Vorkenntnisse der Studierenden sind in Abhängigkeit von der Studienrichtung teilweise verschieden und der Lehrumfang, den die Studierenden des Maschinenbaus über die Dauer von zwei Semestern erlernen, muss in stark gekürzter Form in nur einem Semester vermittelt werden. Daraus ergibt sich vor allem für die Übungen die Notwendigkeit, das Vorgehen zum Lösen der Aufgaben systematisch zu lehren.

Die Studierenden der Studienrichtung Allgemeiner und Konstruktiver Maschinenbau belegen im 5. Semester das Modul "Mechanische
Antriebe". In dem Modul sind mit der Prüfungsordnung 2019 das Fach Antriebselemente und der Konstruktionsbeleg zusammengefasst. Im Rahmen von wöchentlichen Vorlesungen und 6 Übungen erlernen die Studierenden im Fach Antriebselemente die Grundlagen zur Gestaltung, Auslegung und Berechnung von Umlaufrädergetrieben, speziellen hochübersetzenden Getrieben, mehrstufigen Getrieben, Hüllgetrieben, stufenlosen Getrieben und Schaltgetrieben (Abb. 4). Die zweite Modulleistung erlangen die Studierenden durch die Anfertigung einer Belegarbeit, in der sie ein komplexes Antriebssystem dimensionieren und konstruktiv umsetzen. Die wöchentlichen Konsultationen dienen zur Belegausgabe, der Vermittlung des Vorgehens zur Bearbeitung und der individuellen Diskussion von Lösungsansätzen mit den Studierenden.

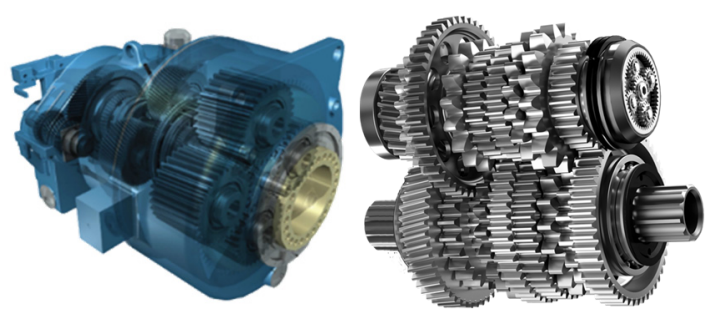

Abb. 4: Stirn- und Planetenradgetriebe

Ergänzend bietet der Lehrstuhl den Studierenden des 5. Semesters in den Modulen „Erweiterte Grundlagen im Maschinenbau" und "Grundlagen der Verbrennungsmotoren und Antriebssysteme" die Vorlesung Antriebssysteme an. In den wöchentlichen Veranstaltungen werden das Zusammenwirken von Anund Abtriebsmaschinen, die Eigenschaften und Einsatzmöglichkeiten unterschiedlicher Kupplungsarten und die Möglichkeiten der Schwingungsanalyse den Studierenden vermittelt (Abb. 5). Die Anwendbarkeit des im Studium bereits erlernten Wissens auf verschiedene Fragestellungen der Antriebstechnik und die Möglichkeiten der Dimensionierung von Antriebsstrangkomponenten mit Hilfe händischer Berechnungen werden den Studierenden in Vorrechenübungen während der regulären Vorlesungszeiten vorgestellt. Neben der Betreuung der Studierenden in den aufgeführten Lehrveranstaltungen begleiten die Mitarbeiter des Lehrstuhls im Jahr durchschnittlich 
20 Projektarbeiten im Fachpraktikum, 10 Projektarbeiten im Forschungspraktikum und 20 Diplomarbeiten.

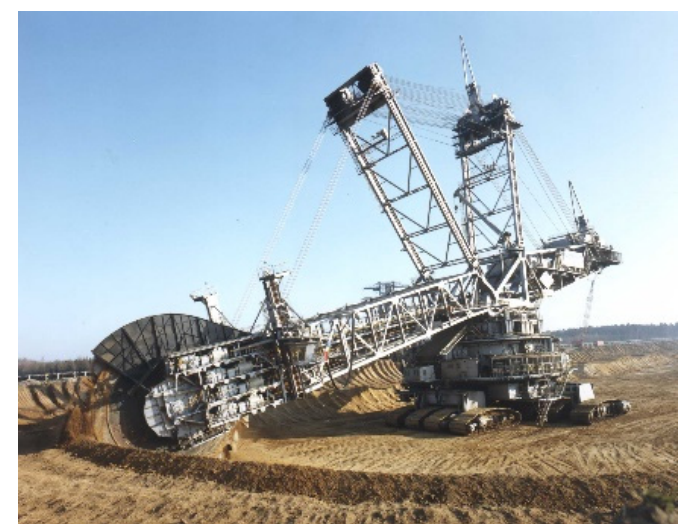

Abb. 5: „Antriebssystem" Schaufelradbagger

\section{Online-Lehre über drei Semester}

Im Winter- und Sommersemester werden durch den Lehrstuhl Maschinenelemente im regulären Lehrbetrieb Vorlesungen im Umfang von 9 bzw. 11 Semesterwochenstunden und die Übungsbetreuung in 33 bzw. 29 Semesterwochenstunden angeboten. Mit Rücksicht auf die zeitlichen und technischen Möglichkeiten der Studentinnen und Studenten und mit dem Ziel, den vollumfänglichen Lehrinhalt in allen Kursen anzubieten, werden im Laufe der Semester alle Materialien zu den Lehrveranstaltungen rechtzeitig über OPAL zur Verfügung gestellt. Informationen zu den Lehrveranstaltungen werden vor jeder Vorlesungswoche über OPAL an die Studierenden gesendet und stehen über das gesamte Semester auch im OPAL zur Verfügung. Die Durchführung der Lehre unter den geänderten Randbedingungen soll im Folgenden für die verschiedenen Lehrveranstaltungen näher erläutert werden.

Für die Vermittlung des Lehrinhaltes im Rahmen der Vorlesung Maschinenelemente und Antriebssysteme ist es sehr hilfreich, dass alle Vorlesungsunterlagen bereits als PowerPoint Folien vorliegen und damit die Grundlage für die digitale Lehre in diesen Fächern bilden können. In der Vorlesung im Fach Antriebselemente dienen die handschriftlichen Skripte aus dem Wintersemester 2019/20 als Vorlage. In Abhängigkeit vom Inhalt der Veranstaltung sind Abschnitte in unterschiedlichem Umfang in der Vorlage nicht enthalten, um ein besseres
Verständnis für komplizierte Sachverhalte durch ein selbstständiges Mitschreiben zu erzielen. Diese Mitschriften werden den Studierenden vor der Vorlesung zur Verfügung gestellt und in den Veranstaltungen dann die bereits vorhandenen Passagen präsentiert, diskutiert und fehlende Formeln, Diagramme und Texte ergänzt. Neben den Mitschriften werden Sachverhalte mit Hilfe von Modellen und einem Visualizer sowie durch Videos und Präsentationen erläutert (Abb. 6 bis 8). Im Gegensatz zum Sommersemester 2020, in dem die Vorlesungen als vertonte PowerPoint Präsentationen über OPAL zur Verfügung gestellt wurden, finden seit dem Wintersemester 2020/21 alle Vorlesungen in GoToMeeting mit dem Videobild des jeweiligen Dozenten statt. Die Aufzeichnung der Lehrveranstaltungen kann direkt in GoToMeeting komfortabel und gut komprimiert erfolgen. Die Videos zu den Vorlesungen werden nach der Konvertierung im VideoCampus im Laufe der aktuellen Woche im OPAL veröffentlicht. Die Folien zu den Vorlesungen können als PDF-Datei im OPAL zusätzlich heruntergeladen werden.

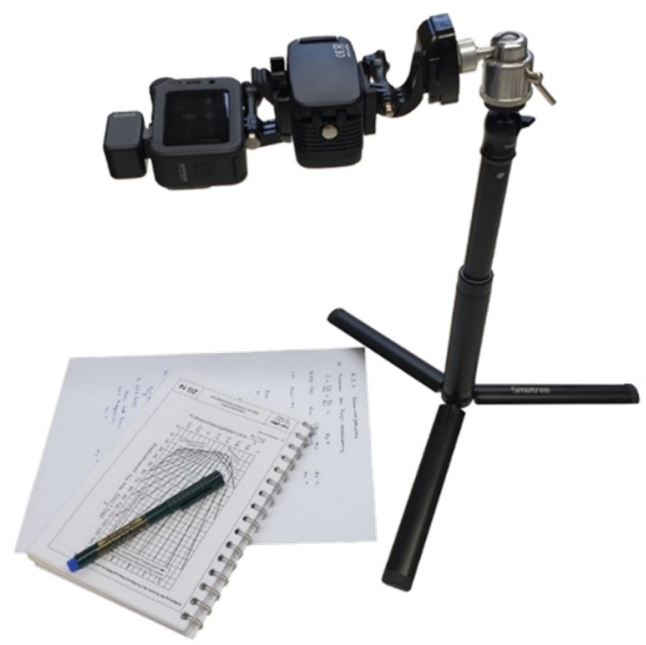

Abb. 6: Aufzeichnung der Übungsanleitung im Sommersemester 2020

Die Anzahl der Teilnehmer während der regulären Veranstaltungszeiten entspricht etwa 30 bis $50 \%$ der im OPAL eingeschriebenen Studierenden, jedoch werden die Mitschnitte der Vorlesungen im VideoCampus intensiv genutzt. Fragen und Hinweise während der Vorlesungen über den Chat sind eher selten, ausgenommen es treten technische Schwierigkeiten auf. Vor allem zu Beginn 2021 war dies bei 
der Nutzung von GoToMeeting der Fall. Verbindungsabbrüche, schlechte Ton- und auch Bildqualität erlaubten zeitweise keinen reibungslosen Ablauf der Lehrveranstaltung. Zum Ende des Semesters und im Sommersemester traten die beschriebenen Probleme aber nur noch sporadisch auf und störten den Lehrbetrieb kaum.

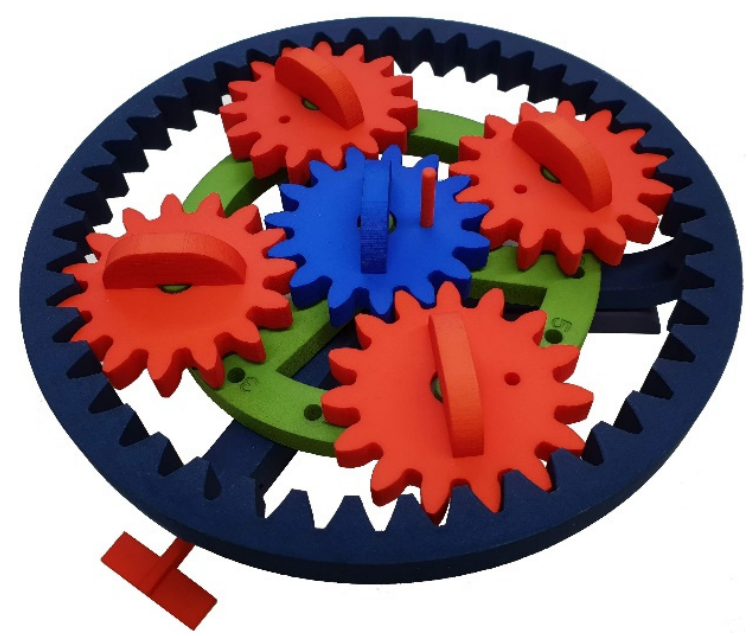

Abb. 7: Modell zur Veranschaulichung unterschiedlicher Planetenradgetriebe

Zum selbständigen Bearbeiten der Übungsaufgaben im Fach Maschinenelemente wird den Studierenden zu Beginn jeder Woche ein Video bereitgestellt, in dem Schritt für Schritt das Vorgehen zum Lösen der Fragestellung erläutert ist. Die im Sommersemester zur Aufzeichnung genutzte und als Visualizer umfunktionierte Videokamera inklusive Beleuchtung wurde durch ein beschreibbares Convertible ersetzt und die Videos mit OBS aufgezeichnet (Abb. 6). Die Nachbearbeitung der Videos dient zur Beseitigung von Versprechern und ermöglicht den zeitlichen Umfang der Anleitung einzukürzen. In den Konsultationen zum Fach Maschinenelemente werden die Fragen zu den Anleitungsvideos durch mehrere Mitarbeiter und Tutoren zu den regulären Veranstaltungszeiten beantwortet.

Die Studierenden hoben die lockere Stimmung in den Konsultationen bei gleichzeitig guter fachlicher Betreuung hervor, kamen mit den Videoanleitungen großteils gut zurecht, wünschten sich aber auch die Bereitstellung der Videos bereits vor der eigentlichen Vorlesungswoche, individuellere Betreuung in kleineren Gruppen und das Hintergrundwissen zur Übung bereits in der Vorlesung gehört zu haben.

Zur Übung im Fach Antriebselemente werden ebenfalls Anleitungsvideos und Konsultationen angeboten. In der ersten Hälfte der Konsultation haben die Studierenden Zeit, sich das Anleitungsvideo anzuschauen und dabei bereits Ihre Fragen zu stellen. Im zweiten Teil werden die Lösungen zu den Aufgaben vorgestellt und weitere Fragen zum Lösungsweg diskutiert. Die digitale Umsetzung der Lehre im Fach Antriebselemente wurde von den Studierenden positiv bewertet, gleichzeitig aber auch der Wunsch geäußert, Unterlagen und Videos mit mehr Vorlaufzeit herunterladen zu können.

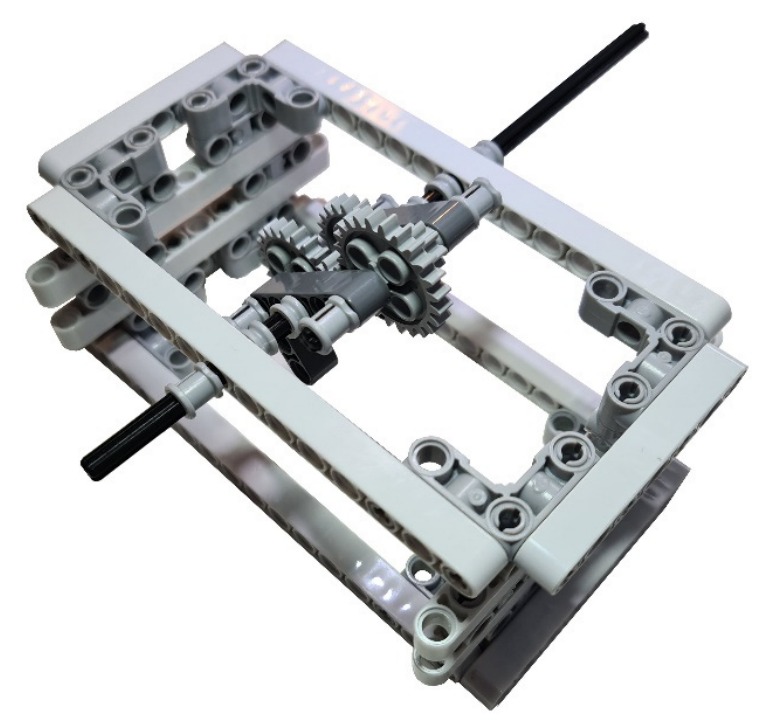

Abb. 8: Modell zur Erläuterung der Selbsthemmung

Zum Konstruktionsbeleg und zu den Belegen in Maschinenelemente und Konstruktionslehre werden ebenfalls Konsultationen angeboten und im OPAL jeweils Foren zur Diskussion von Fragen zum Beleg eingerichtet. Auch wenn eine höhere Teilnehmerzahl wünschenswert und zum Erreichen besserer Ergebnisse sicher hilfreich wäre, werden zu den Abgabeterminen verhältnismäßig viele Belege abgegeben.

\section{Leistungskontrolle und Prüfungen in Präsenz und Online}

Am Ende des Sommersemesters werden im Rahmen der Übung und zur Vorbereitung der Prüfung die vermittelten Lehrinhalte durch 
eine Leistungskontrolle abgefragt. Die Prüfung mit einer Dauer von 4 Stunden besteht aus zwei Teilen. In den ersten zwei Stunden müssen die Studierenden ohne Unterlagen eine technische Zeichnung anfertigen und Fragen zum Lehrinhalt beantworten. Im zweiten Teil der Prüfung können dann alle Unterlagen zum Lösen der gestellten Berechnungsaufgaben verwendet werden.

Nach anfänglichen Bedenken gegenüber einer digitalen Durchführung der Leistungskontrolle und Prüfung und den ersten Versuchen, einen Test in OPAL aufzusetzen, waren vor allem die Möglichkeiten der automatisierten Korrektur ausschlaggebend, die Leistungskontrolle digital durchzuführen. Durch die Beteiligung aller Mitarbeiter bei der Formulierung der Fragen, entstand eine umfangreiche Aufgabensammlung, die als Grundlage für die Erstellung der Leistungskontrolle diente. Bei der Durchführung des Tests zum ersten Termin im Sommersemester 2020 ergaben sich vielfältige Probleme. Diese waren zum Teil auf die Überlastung des Systems, aber auch auf fehlerhafte Einstellungen und die erteilten Zugangsberechtigungen zurückzuführen. Die zweite Leistungskontrolle verlief nach den gesammelten Erfahrungen des ersten Versuches nahezu problemlos, so dass die Vorbereitungen zur digitalen Prüfung im Sommersemester 2020 anliefen.

In der verkürzten dreistündigen Prüfung mussten die Studierenden händisch Zeichnungen erstellen, digitalisieren und in OPALexam hochladen, Fragen beantworten und Berechnungsaufgaben lösen. OPALexam war bei der Durchführung der Prüfung überlastet, eine Bedienung im Browser war nur mit Verzögerungen möglich und Prüfungsleistungen wurden zum Teil nicht abgespeichert. Eine eher misslungene digitale Prüfung beendet damit eine recht gut gelungene Vorlesungszeit im Sommersemester 2020.

Nach den negativen Erfahrungen in der vorangegangenen Prüfungsperiode und den Diskussionen zu gerechten Prüfungsbedingungen während der ersten „Lessons Learned“ Konferenz, war zu Beginn des Wintersemesters 2020/21 die nochmalige Durchführung von Prüfungen in OPALexam nicht vorgesehen. Der Aufwand zur Vorbereitung der Prüfungen und die enormen technischen Schwierigkeiten bei der Durchführung konnten aus damaliger Sicht die Zeitersparnis bei der Bewertung nicht aufwiegen.

Da zum Jahreswechsel nicht abzusehen war, ob eine Durchführung von Präsenzprüfungen überhaupt möglich ist, musste die Entscheidung zur Prüfungsdurchführung dann doch revidiert werden. Mit Hilfe engagierter Mitarbeiter wurden vier digitale Prüfungen mit einer Prüfungsdauer von 60, 90, 120 und $180 \mathrm{~min}$ neu erarbeitet bzw. vorhandene Fragen überarbeitet und konnten fristgerecht dem ZiLL zur Überprüfung freigegeben werden. Zu drei der vier Prüfungen wurden Probeklausuren ohne Terminbindung angeboten. Die Studierenden konnten frei wählen, wann Sie den Test zum Prüfen der eigenen technischen Voraussetzungen und zum Kennenlernen der verschiedenen Antwortmöglichkeiten durchführen. Im Fach Antriebselemente wurde eine Probeklausur zum angebotenen Termin in OPALexam durchgeführt und parallel in BigBlueButton betreut. Sowohl die Art der Fragestellungen in dem Multiple-Choice-Test wie auch die Eingabemöglichkeiten in den Berechnungsaufgaben sollten die Studierenden im Rahmen des Tests kennenlernen. Rückmeldungen zu Problemen bei der Testdurchführung gab es nicht, die Möglichkeit der Probeklausur wurde aber auch nicht von allen eingeschriebenen Studierenden wahrgenommen.

Bei der Durchführung der Prüfungen in OPALexam gab es in der Prüfungsperiode im Wintersemester 2020/21 nahezu keine technischen Schwierigkeiten. Nur wenigen Studierenden musste nach Verbindungsproblemen ein Neustart des Tests ermöglicht werden. Die Betreuung der Prüfung erfolgte in kleineren Gruppen mit Hilfe von BigBlueButton und im Notfall über Telefon. Beschwerden zur Prüfung gab es bzgl. der verfügbaren Zeit zur Bearbeitung von Fragen. Diese reichte nicht aus, um alle Fragen in den Unterlagen nachzuschlagen. Eine Vielzahl von Studierenden, die bereits im letzten Semester Erfahrungen mit digitalen Prüfungen sammeln konnten, schienen aber gut mit den Aufgaben zurechtzukommen, das Hochladen von Zeichnungen funktionierte gut und die Notenverteilung ist mit Prüfungen in Präsenz vergleichbar. 